\title{
Carnets
}

Revue électronique d'études françaises de l'APEF

Deuxième série - $11 \mid 2017$

Les écrivains écrivent l'Europe en français

\section{Regards exotopiques sur deux portes de l'Europe : la crise migratoire à Lampedusa et à Mayotte dans Eldorado et Tropique de la violence}

\section{Silvia U. Baage}

\section{(2) OpenEdition}

Journals

Édition électronique

URL : http://journals.openedition.org/carnets/2369

DOI : $10.4000 /$ carnets.2369

ISSN : 1646-7698

Éditeur

APEF

\section{Référence électronique}

Silvia U. Baage, « Regards exotopiques sur deux portes de l'Europe : la crise migratoire à Lampedusa et à Mayotte dans Eldorado et Tropique de la violence », Carnets [En ligne], Deuxième série - 11 | 2017, mis en ligne le 30 novembre 2017, consulté le 10 décembre 2020. URL : http:// journals.openedition.org/carnets/2369; DOI : https://doi.org/10.4000/carnets.2369

Ce document a été généré automatiquement le 10 décembre 2020.

\section{(c) (1) (8)}

Carnets est mis à disposition selon les termes de la licence Creative Commons - Atribution - Pas d'utilisation commerciale 4.0 International. 


\title{
Regards exotopiques sur deux portes de l'Europe : la crise migratoire à Lampedusa et à Mayotte dans Eldorado et Tropique de la violence
}

\author{
Silvia U. Baage
}

1 Avec 25,000 naufragés au cours de 20 ans, la Méditerranée s'est convertie en cimetière pour les migrants clandestins ayant échoué l'approche du Vieux Continent par la voie maritime. Les drames aux larges des côtes d'une île italienne, Lampedusa, montrent de façon évidente le désespoir des milliers de migrants qui risquent tout afin d'atteindre une des portes de l'Europe. Si l'Europe reste un Eldorado rêvé pour les nouveaux damnés de la terre, elle se transforme de plus en plus en forteresse, à en croire Yasmina Lahlou (Lahlou, 2015 : 2-3). Que reste-t-il alors des progrès civilisationnels ? Et quel est l'impact sur la production littéraire d'expression française, entre autres, celle de Fatou Diome, écrivaine d'origine sénégalaise installée à Strasbourg ? En 2017, lors de la campagne des élections présidentielles, cette dernière dénonce avec véhémence le discours populiste de l'extrême droite : « Messagers du malheur, toujours à trier, accuser, rejeter, ils sont prêts à sonner la curée ; je ne serai pas de ceux qui auront laissé les loups dévorer les agneaux au nom de l'identité nationale. Marianne porte plainte » (Diome, 2017 : 7).

2 Mais la crise migratoire est un phénomène international qui s'étend jusqu'à la France lointaine, notamment à Mayotte dans l'océan Indien. Quoiqu'elle y reste moins visible, elle présente de nombreux défis qui concernent les relations nord-sud ainsi que les fondements de l'Union européenne, comme l'indiquent bien Adrien Boschet et JeanBaptiste Guégan (2017). C'est dans cet esprit que nous nous pencherons sur deux romans transnationaux qui s'inspirent de l'approche européenne de la crise migratoire, enjeu socio-politique et économique de grande envergure à Lampedusa ainsi qu'à Mayotte. Il s'agira d'analyser la représentation littéraire de ce que l'on pourrait appeler une " 
impasse européenne " face à ces mouvements migratoires clandestins vers ces portes insulaires de l'Europe (Boschet et Guégan, 2017: 75). Dans Eldorado (2006), l'auteur français Laurent Gaudé met en scène deux mouvements migratoires qui s'entrecroisent. Le premier est celui du Syrien Soleiman vers le Nord car Soleiman tente d'atteindre l'Europe tandis que le commandant d'une frégate italienne quitte l'Europe pour se diriger vers l'Afrique du Nord pour faire le chemin des clandestins à l'envers. En revanche, Tropique de la violence (2016) est un roman-témoignage de la Franco-Mauricienne Nathacha Appanah qui prend comme point de départ le flux migratoire vers une porte de l'Europe lointaine, Mayotte, destination privilégiée des Comoriens qui risquent la traversée périlleuse en kwassa-kwassa pour atteindre la France lointaine. Dans ce roman, l'intrigue se concentre sur le destin d'un adolescent, Moïse, abandonné par sa mère qui arrive à Mayotte sans papiers juste après l'accouchement.

Ces deux romans transnationaux sont liés sur le plan thématique, l'écriture de l'Europe, mais aussi par le biais d'une structure polyphonique assez complexe qui nous intéresse ici. Nous nous proposerons donc de comparer l'imagination exotopique, au sens où Bakhtine entend ce terme (Bakhtine, 1984 : 348), en nous focalisant sur le récit de cinq personnages morts et vivants, à la fois oppresseurs et opprimés, chez Appanah, contrairement à Gaudé qui crée deux intrigues qui s'entrecroisent en Afrique du Nord, loin de Lampedusa, pour narrer le périple d'un clandestin et celui d'un " gardien de la forteresse » qui finit par quitter son poste.

\section{Regards exotopiques sur Lampedusa et Mayotte}

4 L'imagination exotopique véhicule une approche de l'espace conçu par de multiples regards qui s'entrecroisent, se multiplient et se contredisent. Dans les romans en question, cette juxtaposition de voix mélangeant le sacré et le profane, phénomène cher à Bakhtine, rend compte de multiples perspectives pour non seulement décrire mais aussi, pour jeter un regard critique sur deux espaces insulaires difficiles, à savoir, deux portes de l'Europe : «l'Europe est ainsi la région du monde où l'on meurt le plus en tentant de franchir une frontière illégalement » (Boschet et Guégan, 2017 : 74).

Dans Eldorado, le récit du commandant Salvatore Piracci confirme ce fait tragique pour Lampedusa, une île italienne qui se trouve sur la route des peuples démunis migrant vers le Nord. De fait, cette île voit de nombreux clandestins qui échouent au large de ses côtes, avalés par la mer ou interceptés par Piracci lors de ses patrouilles entre Lampedusa et Catane. À la suite d'une retrouvaille bouleversante avec une femme rescapée en 2004, il ne supporte plus « le silence (...). La misère (...) face à lui » (Gaudé, $2006: 15)$ et il décide de quitter cette « silhouette noire » (idem : 146). C'est alors loin du « rocher qu'ils rêvaient tous d'atteindre" (idem) que Piracci croise la route de Soleiman, réfugié d'origine syrienne en route vers Ceuta, autre porte maritime de l'Europe ${ }^{1}$. Soleiman ne passera pas par Lampedusa, or son passage par Ceuta, au Maroc, est évocateur du drame humain qui se joue au large d'une des nombreuses portes de l'Europe. Le lecteur ne connaîtra pas la suite de son périple sur le Vieux Continent mais la structure polyphonique du récit permet de découvrir la source de ses forces dans la mystérieuse présence de Massambalo, " le dieu des immigrés » (idem : 208) qui fait qu'ils ont « [l]'Eldorado (...) au fond des yeux. (...) En cela, ils ont été plus riches (...) Qui rêvent toujours plus loin» (idem : 120). Ce personnage mystérieux est bien évidemment Piracci qui se propose de personnifier ce dieu. 
6 La particularité du récit polyphonique de Gaudé consiste à créer un contraste entre le mythe entretenu par les clandestins et les passeurs, celui de l'Eldorado qui s'oppose de façon dramatique à la réalité. Le trajet hautement dangereux finit souvent par mal tourner face au "continent d'eau» (idem:72) d'une mer féroce et sauvage. Cette description est dédoublée dans le récit du commandant lorsqu'il raconte son quotidien sur la mer aussi bien qu'au moment de la rencontre avec une ancienne rescapée d'un navire intercepté qui s'achève sur la mort de son bébé dans ses bras. Dans ce sens, le commandant est à la fois « un visage de ce continent tant espéré » (idem :18), mais aussi le coupable, "le mauvais œil qui traque les désespérés» (idem:67), si bien qu'il doit repousser «les hommes qu'il enviait» (idem:118). Ce contraste d'images renvoie au propos du sociologue Pierre Bourdieu qui prône la juxtaposition de multiples voix afin de mieux cerner les enjeux d'un espace difficile :

(...) il faut substituer aux images simplistes et unilatérales (...) une représentation complexe et multiple, fondée sur l'expression des mêmes réalités dans des discours différents (...) abandonner le point de vue unique, central, dominant (...) au profit de la pluralité des perspectives correspondant à la pluralité des points de vue coexistants et parfois directement concurrents (Bourdieu, 1993 : 14).

7 Il en est résulte « l'affrontement des visions du monde différentes ou antagonistes » (idem :13) et c'est précisément cet affrontement qui nous intéresse dans le sens où les visions des pays dits Nord et celles des pays dits Sud se dépeignent ici.

Mais dans Tropique de la violence, cet "affrontement des visions" est encore plus complexe. L'intrigue juxtapose des récits de groupes sociaux différents pour décrire diverses formes de souffrance qui sont à la fois apaisées mais aussi exacerbées par le phénomène de migration clandestine vers Mayotte ${ }^{2}$. Le roman d'Appanah prend ainsi la migration clandestine comme cadre mais l'intrigue se concentre essentiellement sur une alternance entre des points de vue de riches et de pauvres, de jeunes et de vieux, d'étrangers et d'indigènes, d'hommes et de femmes, de fonctionnaires et de bénévoles qui décrient tous le coût humain de la misère dans ce petit monde au bout du monde.

Parmi les cinq personnages chargés de la narration, deux sont morts. Le roman s'ouvre in medias res sur le récit de Marie qui raconte sa souffrance en tant que femme qui rêve autant d'avoir un bébé. Ce désir se réalise enfin au moment où une clandestine d'origine comorienne met dans les bras de Marie son bébé maudit ${ }^{3}$ avant de disparaître. Marie élève Moïse comme son fils, l'inscrit à « l'école privée de Pamandzi, là où il n'y a que des métropolitains ou des enfants de Mahorais ayant vécu longtemps en France " (Appanah, 2016 : 26). De fait, ils ne vivent « [p]as loin (...) des cases en tôle, des bangas, où vivent des clandestins, et nous fermons à double tour notre maison, mettons des grilles en fer aux fenêtres et des cadenas à notre portail. Nous avons maintenant un chien » (idem :26). En revanche, le deuxième personnage mort, Bruce, n'occupe pas la même position privilégiée que Marie et son fils Moïse qui vivent en huis clos et mangent à leur faim : « Moi aussi je voudrais que quelqu'un me prépare un bol de céréales, putain des céréales je sais même pas le goût que ça a des céréales » (idem :45). En fait, avant de mourir, Bruce habitait « la colline de Kaweni où le bidonville s'étend comme une pieuvre» (idem: 27). Cette métaphore animalière des fonds de la mer illustre bien l'influence de Bruce qui dirige son royaume, le bidonville surnommé le gaza, comme s'il avait de nombreux tentacules qu'il prolonge dans tous les sens, pour attaquer, se défendre et pour se faire respecter.

10 L'image que donnent les deux personnages morts de l'île est foncièrement négative ; cette description négative est d'ailleurs confirmée par le policier Oliver ainsi que par Stéphane, 
bénévole d'origine française : «le quartier défavorisé de Kaweni (...) c'est un bidonville, c'est un ghetto, un dépotoir, un gouffre, une favela, c'est un immense camp de clandestins à ciel ouvert, c'est une énorme poubelle fumante » (Appanah, 2016: 51). Peu importe la classe sociale, tous les récits mettent en avant la monstruosité de l'île: Marie souligne qu'elle ne juge personne car «ce pays nous broie, ce pays fait de nous des êtres malfaisants, ce pays nous enferme entre ses entrailles et nous ne pouvons plus partir » ( idem : 20) ou encore « ce pays ressemble à une poussière incandescente et (...) il suffira d'un rien pour qu'il s'embrase » (idem: 11). Par conséquent, Moïse finit par tuer Bruce, son bourreau, et au lieu d'accepter la protection de la police, il sort de la voiture qui est censée assurer son transfert à la Réunion. Il marche lentement vers « ce bleu magnifique, brillant, ce bleu qui peut-être n'existe qu'ici dans cet océan » (idem : 175) afin de plonger et ne plus remonter. Il meurt comme de nombreux clandestins qui se noient. Comment peut-on donc expliquer la crise humanitaire dans ces deux « lieux dits 'difficiles' [... qui] sont d'abord difficiles à décrire et à penser » (Bourdieu, 1993 : 14) bien qu'ils fassent partie de l'Union européenne? Que se passe-t-il à l'ombre d'une grande civilisation en fleurs?

\section{Le rêve d'Europe: les ombres d'une civilisation en fleurs}

11 Dans un premier temps, Lampedusa et Mayotte représentent le seuil du Vieux Continent ; franchir ce seuil est la promesse d'une vie plus facile pour les peuples démunis en quête de progrès. Le rapprochement à la fois symbolique et géographique de ces deux portes de l'Europe est d'ailleurs signalé de manière évidente dans Tropique de la violence lorsque le policier Olivier constate que «[c]e n'est peut-être qu'une vieille histoire, cent fois entendue, cent fois ressassée. L'histoire d'un pays qui brille de mille feux et que tout le monde veut rejoindre. Il y a des mots pour ça : eldorado, mirage, paradis, chimère, utopie, Lampedusa » (Appanah, 2016:53). Pour les peuples défavorisés, atteindre cet eldorado à Mayotte ou à Lampedusa, est bel et bien un enjeu géopolitique, comme l'explique bien Marie pour les Comoriens à Mayotte où « tous ces miséreux (...) attendent leurs papiers et tous les autres (...) attendent des soins médicaux » (idem: 17). La description de Marie est prégnante de sens, lorsqu'elle traverse deux files d'attente devant la préfecture et le dispensaire où

[1]e drapeau bleu blanc rouge flotte haut. Devant la grille fermée, il est encore temps d'espérer décrocher un ticket qui permette de voir un agent et, enfin, expliquer son cas, sa vie, le pourquoi du comment, déposer son dossier de demande de permis de séjour, réclamer un récépissé, s'enquérir d'une carte de séjour, espérer un renouvellement, une écoute, un sursis, un sésame (Appanah, 2016 : 14-15).

Mais de façon évidente, ni Lampedusa ni Mayotte ne protègent véritablement les peuples démunis. Ces terres italienne et française font partie du Vieux Continent, cette terre d'accueil pour « la communauté des hommes déchus par la peur et l'urgence » (Gaudé, 2006 : 159), pour reprendre les mots de Soleiman dans Eldorado. Or, le Vieux Continent est stupéfait par l'arrivée de "ces embarcations de misère (...) La mer leur apportait régulièrement des corps morts (...) Ces hommes dont ils ne savaient rien, ni le nom, ni le pays, ni l'histoire, venaient s'échouer chez eux et leur cadavre ne pourrait jamais être rendu à leur mère » (idem: 119). Au début, Lampedusa accueille les clandestins noyés de façon posthume au " cimetière d'Eldorado » mais l'île semble se lasser, au fur et à mesure que le nombre augmente, si bien que le commandant constate que l'« hospitalité des gens 
de Lampedusa s'était usée comme son propre regard (...). [L]ui aussi, à trop croiser la misère, (...) avait (...) fini par assécher son humanité » (idem : 120). Cette constatation ne rassure point le commandant et il finit par quitter cette île. En Afrique, il se voit obligé de raconter la vérité sombre

Il parla de la misère des riches. De la vie d'esclave qui attendait la plupart de ceux qui tentaient le voyage. Il parla de l'écœurement devant ces magasins immenses où tout peut s'acheter mais où rien n'est vraiment nécessaire. Il parla de l'argent. De sa violence et de son règne (Gaudé, 2006 : 204).

Mais les avertissements du commandant sont mal reçus par les migrants qui préfèrent « continuer à caresser leur rêve d'Europe avec délices » (idem : 204-205) si bien qu'ils le chassent.

Ce désir d'aller à l'encontre du mythe est plus ambigu dans Tropique de la violence. Stéphane a beau insister sur le contraste entre les beaux paysages magnifiques qui font oublier « la lie, (...) la violence, (...) la fange » (Appanah, 2016 : 111) car « c'est la France ici quand même! » (idem). Comme de nombreux étrangers d'origine européenne, il a du mal à accepter le contraste énorme entre la France métropolitaine et Mayotte et pour se rassurer, il nie ce que Jean Baudrillard appelait la transparence du mal, ou bien, une accélération «dans le vide, (...) c'est par cette anticipation de tous les résultats, par la disponibilité de tous les signes, de toutes les formes, de tous les désirs (...) il faut paradoxalement continuer de vivre comme si [toutes les utopies réalisées] ne l'étaient pas » (Baudrillard, $1990: 11-12$ ). Ce genre de refoulement se manifeste dans l'indifférence devant de nombreuses «formes contemporaines du Mal» (idem: 88) ainsi que dans l'« indistinction des signes» dans le domaine transéconomique ou transpolitique où, selon Baudrillard, « la force de dire le Mal (...) nous a échappé » (idem : 88). En effet, pour l'intrigue qui se déroule à Mayotte, le tague « en vert GAZA » (Appanah, 2016:111) sur le mur du bidonville est aperçu par les nouveaux arrivants étrangers comme «la bonne blague» (idem: 113) plutôt qu'un signe inquiétant d'une instabilité. En revanche, le policier Olivier est plus conscient de l'opacité du mal si bien qu'il se propose d'une part, d'appliquer «la loi française sur une île oubliée » (idem: 53) et que d'autre part, il confirme qu'il se passe des événements inquiétants voire irréels dans cette partie de la France lointaine : «Gaza c'est un no man's land violent où les bandes de gamins shootés au chimique font la foi. Gaza c'est Cape Town, c'est Calcutta, c'est Rio. Gaza c'est Mayotte, Gaza c'est la France » (idem : 51) et au moment du meurtre de Bruce, il déclare : «Voilà, ça va être la guerre à Mayotte » (idem: 51). En ce sens, les regards exotopiques permettent de relever les différentes formes du «langage du Mal [qui] déclenche un tel accès de faiblesse des cultures occidentales, en dépit des pétitions d'intellectuels » (Baudrillard, 1990 : 90).

15 En effet, dans la description de Bruce, le chef du gaza, nous constatons un discours amer au sujet du «bouffon blanc (...) il soûle avec ses paroles et ses matchs de foot » (Appanah, 2016 : 131). De plus, quand il s'agit de son île, le discours de Bruce manifeste la «force de dire le Mal » (Baudrillard, 1990 : 88), contrairement aux autres personnages qui nient ce que Bruce dit de façon explicite :

C'est Mayotte ici et toi tu dis c'est la France. Va chier ! La France c'est comme ça ? En France tu vois des enfants traîner du matin au soir comme ça, toi ? En France il y a des kwassas qui arrivent par dizaines comme ça avec des gens qui débarquent sur les plages et certains sont déjà à demi morts? En France les gens mettent des grilles de fer à leurs fenêtres comme ça ? En France les gens chient et jettent leurs ordures dans les ravines comme ça? (Appanah, 2016:97) 
Si les propos de Bruce mettent en cause le glissement du signifiant et du signifié, la France voire l'Europe, dans le contexte de la France d'outre-mer, ils nous renvoient également aux connotations et dénotations du terme "ombre", présentes tout au long des deux textes à travers la métaphore filée de l'ombre. Mayotte représente cette dimension symbolique à laquelle renvoie le Dictionnaire des symboles car cette île est une zone sombre « qui s'oppose à la lumière » (Chevalier et Gheerbrant, $1982: 700$ ), ou pour aller encore plus loin, Mayotte est un espace où se manifeste «l'image des choses fugitives, irréelles, changeantes» (idem: 700) comme nous venons de le voir dans les descriptions des Occidentaux si bien que Moïse, en prison, ne veut plus avoir « rien à faire avec ce genre de misère. (...) ceux de dehors, le ventre vide, qui (...) deviennent fous au parfum bleu du poulet qui cuit » (Appanah, $2016: 38$ ). En outre, les personnages Bruce et Marie sont morts et s'adressent au lecteur, voire aux personnages, c'est le cas de Marie qui cherche à calmer Moïse dans sa cellule : « Je me dis que mes paroles de morte peuvent se mêler aux vapeurs de ses rêves et qu'en se réveillant pour de bon, tout à l'heure, il pourra peut-être s'en souvenir » (idem: 56 ). Dans l'ensemble, le récit fait connaitre les coins sombres d'une île tropicale à la fois pour les riches et pour les pauvres.

Dans Eldorado, ce symbolisme est encore plus frappant: le commandant explique sa décision de partir en insistant sur « [1]a fatigue se sa propre existence [qui] lui collait à la peau » (Gaudé, 2006 : 119). À Lampedusa, il n'arrive plus à assurer ses responsabilités en tant que gardien des «portes de la citadelle. [... Être] la muraille de l'Europe » (idem: 67). Il ne faut pas non plus oublier Soleiman qui est bien conscient du fait qu'il se transforme en ombre ${ }^{4}$ au moment où il commence le voyage vers l'Eldorado, jusqu'à ce qu'il croise le commandant en forme d'«ombre de Massambalo» (idem: 227), qui, selon le conteur ivoirien, « vit quelque part en Afrique, terré dans un trou (...). Il a des esprits qui voyagent pour lui. (...) [Ils] sillonnent le continent. (...) C'est au voyageur qui les croise de deviner leur identité " (Gaudé, 2006: 208). En effet, dans le contexte de l'Afrique, se présente visiblement la troisième dimension du terme «ombre » en tant que « la seconde nature des êtres et des choses liée à la mort » (Chevalier et Gheerbrant, 1982: 701) car le destin du commandant s'achève, une fois que Soleiman aura fait son offrande à celui qu'il croit être une des « ombres de Massambalo » (Gaudé, $2006: 234$ ). Il est renversé sur la route par un camion chargé d'hommes « qui s'élançaient à la conquête des frontières » (idem : 238). Ce lien avec la mort, dans le contexte de l'écriture de l'Europe, soulève sans conteste la question des affinités: d'une part, l'Europe, voire le Nord, vit "à l'ombre des excroissances » comme dirait Baudrillard, mais d'autre part, ce dernier insiste sur «le privilège des phénomènes extrêmes, et de la catastrophe en général (...). L'ordre secret de la catastrophe, c'est l'affinité de tous ces processus entre eux " (Baudrillard, 1990: 74). Quels sont donc les objectifs des écrivains transnationaux tels que Gaudé et Appanah qui s'intéressent à cette (in)cohérence des phénomènes inquiétants dans une Europe à la fois proche et lointaine?

\section{Écrire l'Europe : la cohérence des phénomènes extrêmes ou les taches sur la robe de princesse des Lumières $^{5}$}

18 À l'heure actuelle, la migration clandestine est sans aucun doute un des phénomènes les plus inquiétants en Méditerranée. Comme nous l'avons déjà évoqué, cette crise se 
manifeste globalement. Or, dans Tropique de la violence, le personnage d'Olivier fait une constatation triste au sujet de l'invisibilité de la crise migratoire dans l'océan Indien car il existe, selon lui, une affinité entre la crise migratoire en Méditerranée et celle dans l'océan Indien :

Il m'est arrivé d'espérer quand il y a eu le petit Syrien échoué sur une plage turque. Je me suis dit que quelqu'un, quelque part, se souviendrait de cette île française et dirait qu'ici aussi les enfants meurent sur les plages. (...) Pourtant, il n'y a jamais rien qui change et parfois j'ai l'impression de vivre dans une dimension parallèle où ce qui se passe ici ne traverse jamais l'océan. (...) Les vies sur cette terre valent autant que toutes les vies sur les autres terres, n'est-ce pas? (Appanah, 2016:52)

Il en va de même pour l'autre roman; dans Eldorado, le commandant fait une remarque similaire lorsqu'il dénonce le coût humain de la crise migratoire en Méditerranée. Il est conscient des enjeux des relations internationales dans ce « combat politique : l'Europe hausse le ton contre la mainmise de la Syrie sur le Liban, en réponse Damas affrète un navire de crève-la-faim qu'il lance à l'assaut de la forteresse européenne. On pourrait presque appeler cela du langage diplomatique » (Gaudé, 2006:33). Si cohérence il y a, la présence de ce genre de phénomènes extrêmes montre l'impuissance des gardiens de cette forteresse, l'Europe. Or, comme l'indiquent de nombreuses voix dans Tropique de la violence, en ce qui concerne les stratégies de défense, elles ne restent qu'une illusion qui, selon Baudrillard, aboutit à un échec. Cet échec confirmerait le "vide politique » tout en produisant un autre résultat inquiétant : «[i]l relève d'une autre logique encore, qui est l'initiative de l'inversion des rôles: des spectateurs (...) se font acteurs» (Baudrillard, 1990 : 83). Nous regarderons de plus près dans la dernière partie des exemples précis de cette inversion des rôles mais retenons ici les effets sur l'ensemble. La migration est un phénomène récurrent à travers les siècles, à en croire Hervé Le Bras qui souligne que « les invasions qui impressionnent tant nos contemporains datent d'une époque plus récente et parfois d'interprétations historiques erronées nourries par des biais idéologiques » (Le Bras, 2017 : 22). C'est dans ce sens qu'il faut comprendre l'image de la citadelle-Europe ou de la forteresse-Europe car le mouvement de la vague migratoire est souvent perçu, de l'intérieur, comme une invasion du continent européen qu'il faut absolument arrêter, au lieu de souligner la présence d'un drame voire d'une tragédie humaine, pour reprendre le vocabulaire de l'écrivaine franco-sénégalaise Fatou Diome, partisane d'une approche plus humanitaire, à l'encontre des discours populistes. Et cette approche humanitaire miserait sur «l'enseignement des humanités» (Diome, 2017: 134) afin de contrer la peur de l'autre.

20 En effet, dans son essai Marianne porte plainte, Fatou Diome souligne qu'il est essentiel de «[r]elire et faire lire des grands penseurs qui étayent la culture française, voilà le remède contre le déclinisme et la xénophobie des théoriciens du choc des civilisations " (Diome, 2017 : 136). Dans un monde de plus en plus globalisé, il convient de diversifier et de juxtaposer des images antithétiques. Ces idées n'étant pas incompatibles, elles s'inspiraient plutôt du modèle de Montesquieu, «ce phare (...) assez grand pour guider toutes les barques voguant vers l'humain» (idem: 135). Selon Diome, il est question de contrarier les fausses représentations de menaces de l'identité française, principalement à cause des effets négatifs de la crise migratoire :

Les populistes fondent leur offre politique sur l'archaïque peur de l'autre, Montesquieu soulignait déjà au XVIII ${ }^{\mathrm{e}}$ siècle les vertus de la rencontre : l'éveil à soi passe par une connaissance des autres, qui elle-même mène à la conscience d'une humanité commune. (...) L'auteur des Lettres persanes cultiva, non le nombrilisme, mais la distanciation, empruntant des yeux persans pour croquer ses 
contemporains avec empathie. Réunissant Occidentaux et Orientaux, ironisant pareillement sur leurs mœurs, il savait ce que la réflexivité du regard ôte à l'animosité, pour instaurer l'égalité et le respect mutuel, préalables à toute fraternité (Diome, $2017: 135)$.

21 C'est précisément la question du respect mutuel et de la fraternité qui est en jeu dans ces deux romans polyphoniques, Eldorado et Tropique de la violence, car ils juxtaposent tous les deux de nombreuses voix, comme nous l'avons montré, afin de relativiser les discours sur deux portes de l'Europe.

Il n'est pas difficile de faire le rapprochement entre l'utilité des regards exotopiques chez Gaudé et Appanah d'une part, et la réflexivité du regard de Montesquieu d'autre part, si bien que dans le contexte de la migration clandestine, le jeu de distanciation et d'identification évoque de nombreuses questions d'ordre moral et éthique. Il s'agit de créer de l'empathie pour les dominants ainsi que pour les dominés, voire pour les gardiens de la citadelle et les démunis. Visiblement, dans Eldorado, le commandant devient le «visage de l'Europe » qui se met à la place des migrants ayant perdu des êtres bien aimés et il se demande s'il faut prêter une arme à la victime du trafic humain pour qu'elle puisse se venger pour la mort de son bébé. S'il s'en veut de ne pas avoir aidé un jeune migrant clandestin qu'il aurait pu cacher dans sa cabine, il finit par attaquer un capitaine libyen afin de soulager sa détresse de ne pas avoir pu sauver trois embarcations manquantes. En outre, de nombreuses digressions ainsi que des passages imaginaires montrent ce genre de combat intérieur du commandant, par exemple, lorsqu'il fait face à son échec: «Les corps noyés passaient peut-être à l'instant même sous la coque de la frégate » (Gaudé, 2006 : 84). C'est ainsi que le commandant décide de faire sonner la sirène « comme un dernier salut. Pour dire qu'ils avaient tout fait pour les trouver et pour s'excuser de n'y être parvenus » (idem). On pourrait donc avancer l'argument que les hésitations du commandant, avec les moments de forces et de faiblesse, renvoient à ce que Diome retient des textes de Montesquieu, c'est-à-dire, l'auteur « rassur[e] Marianne sur sa beauté, tout en soulignant les petites taches qu'elle obstinait à maquiller » (Diome, 2017: 136). Gaudé est loin de créer des moments comiques car il rapproche son personnage de la philosophie existentialiste de Jean-Paul Sartre (1946), pour qui ne pas choisir, c'est encore choisir.

Pour les démunis, la question de l'empathie se pose en termes différents car ils n'ont pas le choix de ne pas choisir. Dans les deux textes, les récits des marginalisés montrent le combat aux bas-fonds où il faut se battre pour survivre quoi qu'il en coûte. Chez Gaudé, ce combat est illustré à travers la description des épreuves de Soleiman qui est obligé d'attaquer un marchand à Ghardaïa afin d'assurer sa survie : "J'ai volé. (...) Je suis une bête charognarde qui sait sentir l'odeur de l'argent comme celle d'une carcasse faisandée » (Gaudé, 2006 : 157). Il regrette son premier acte de violence mais au moment où il est question d'agir pour sauver son ami boiteux, lorsqu'ils passent le seuil de la forteresse-Europe à Ceuta, il retrouve le chemin vers l'humanité : «Si je l'avais laissé accrocher aux barbelés, je n'aurais plus jamais trouvé le sommeil (...). Boubakar le sait bien. C'est pour cela aussi qu'il a tiré de toute sa force pour que le fil barbelé me laisse passer » (idem : 224). Or, dans Tropique de la violence, il n'est pas question de petits gestes ou réflexes pour sauver les compagnons du voyage. Bruce règne avec une violence crue et il ne montre aucune rancune : "Pour rester le chef il faut punir et j'ai puni » (Appanah, 2016 : 96). Dans son récit, ses flashbacks sont présentés comme des arguments pour son mode de vie : "Avant tout, il faut avoir de l'argent, de la thune, du fric, money money money, il faut que ça rentre, il faut que ça sorte, il faut que ça boive, que ça fume et que ça 
revende » (idem : 95). En revanche, Moïse regrette d'avoir tué un homme, Bruce, et il se rend tout de suite. C'est d'ailleurs son histoire personnelle d'enfant adopté qui permet au lecteur de mieux comprendre sa descente aux enfers.

Si les deux textes ont beau souligner le besoin d'humanité et de fraternité face à une crise humanitaire, ils présentent aussi de nombreuses contradictions internes qu'il s'agit de placer dans le contexte des débats actuels au sujet de la mondialisation. Est-il possible de trouver ce que Patrick Savidan nomme une "nouvelle figure de solidarité " (Savidan, 2009 : 33) dans les textes de ces deux écrivains transnationaux d'expression française qui se proposent d'écrire l'Europe ? Afin de répondre à cette question, il convient de jeter un regard critique voire exotopique sur le fossé Nord-Sud.

\section{Le fossé Nord-Sud : regards exotopiques sur la mondialisation}

Dans son analyse controverse intitulée Le Mythe du fossé Nord-Sud ou comment on cultive le sous-développement, le géographe français Yves Montenay propose de s'éloigner des interprétations misérabilistes des tiers-mondistes d'une part, et des arguments des partisans d'une anti-mondialisation, d'autre part. Selon Montenay, il s'agit d'insister sur l'ensemble des progrès civilisationnels car "l'enfer urbain ne découle ni de la colonisation (qui a créé de bons 'noyaux urbains') ou autre 'mauvaise action' du Nord, ni de la croissance économique » (Montenay, 2003 : 148). En effet, Montenay fait référence à l'incurie des gouvernements du tiers-monde qui manqueraient de légitimité voire de responsabilité politique pour assurer l'alimentation et le bien-être de la population: «Mais l'opinion publique occidentale n'est pas informée » (idem: 151). Quant au problème du sous-développement, Montenay remarque qu'il est impossible de

briser l'équilibre de la pauvreté (...). Une réduction de $20 \%$ de la population de tous ces pays nécessiterait le 'déménagement' de $18 \%$ de la population mondiale, donc plus que la population des pays riches. Pour la France cela signifierait recevoir 72 millions d'étrangers supplémentaires » (Montenay, $2003: 87$ ).

$\mathrm{Si}$ ces deux romans transnationaux se penchent sur la crise migratoire dans deux portes insulaires de l'Europe dans le but de critiquer le manque de solidarité du Nord, les auteurs envisagent néanmoins des solutions plus ou moins radicales qui tendent vers l'extrême, comme nous l'avons déjà évoqué dans la dernière partie en ce qui concerne le vide politique, au sens où Baudrillard entend ce terme.

On ne peut pas s'empêcher de remarquer que Gaudé et Appanah créent des intrigues qui aboutissent à l'inversion des rôles dans le sens où les dominés prennent le contrôle, tout en renversant, en quelque sorte, la hiérarchie sociopolitique. Dans Tropique de la violence, ce sont les enfants du bidonville qui s'organisent et mettent en marche un véritable mouvement de résistance, "machettes à main» (Appanah, 2016: 173) à l'instar des esclaves marrons dans les anciennes îles à rhum. Or, le lecteur ne connaîtra pas la suite du destin de ces enfants pourtant exploités et marginalisés car dans l'ensemble, la situation extrême reste difficile à imaginer voire à améliorer, comme l'explique bien Moïse lorsqu'il critique les assistants sociaux et bénévoles occidentaux sur place :

ils croient vraiment que quelques séances de cinéma, quelques matchs de foot ou du pop américain suffiront à nous faire oublier la misère, la crasse et la violence. (...) Il n'y a pas de séance de cinéma ou de match de foot qui pourrait égaler le fait de 
posséder quelque chose, un objet qui ne soit rien qu'à soi, même si ce n'est qu'une vieille brosse à dents (Appanah, $2016: 125)$.

C'est ainsi que le lecteur trouve, à travers l'imaginaire exotopique, de nombreuses voix qui mettent en question l'intervention du Nord. En effet, Stéphane va encore plus loin lorsqu'il critique le caractère paradoxal de l'aide humanitaire: "Je voulais faire autrement, ne plus incarner le cliché de l'humanitaire baroudeur aigri » (idem: 114), ou encore: «des sages-femmes, des infirmiers, des jeunes entrepreneurs, des instituteurs, tous des jeunes comme moi, tous blancs comme moi, des théories plein la bouche et pas une once de courage dans les mains » (idem: 115). Ces propos ne manquent donc pas de renvoyer de nouveau à cette différence radicale dont parle Baudrillard lorsqu'il évoque la misère et les projets humanitaires: «il n'y a pas de bon usage de la différence. (...) Partout l'œcuménisme humanitaire, l'œcuménisme de la différence est en pleine impasse (...) les signes du folklore et de la misère sont de bons opérateurs de la différence " (Baudrillard, 1990 : 137). Il ne s'agit pas d'une incompréhensibilité éternelle au sens où Segalen entend ce terme, mais d'une impossibilité de la rencontre entre le Nord et le Sud qui se manifeste, dans le cas d'Appanah, dans l'impossibilité de trouver un sens dans cette absence de sens de ces phénomènes extrêmes, en l'occurrence, la faim et la misère ${ }^{6}$.

Chez Gaudé, l'inversion des rôles face au vide politique prend une autre forme car c'est le gardien de la forteresse-Europe qui quitte l'Eldorado tandis que les clandestins s'attaquent à la citadelle. La solution au flux migratoire est de créer une sorte de deus ex machina à travers le commandant qui rejoint les pays dits Sud où il reçoit l'offre de passer de l'autre côté, c'est à dire, d'aider les passeurs à trouver un passage vers l'Europe vu qu'il connaît les stratégies des gardiens de la forteresse-Europe. Si ce dernier finit par refuser cette offre, son périple à l'encontre du flux migratoire cache le caractère paradoxal de l'avènement de la mondialisation et des relations Nord-Sud, à en croire Le Bras :

Que des personnages de pays pauvres souhaitent migrer et que les pays riches ne veuillent pas les recevoir est une manifestation traditionnelle d'égoïsme, mais que ces mêmes habitants des pays riches souhaitent migrer en grand nombre alors qu'ils refusent l'entrée des plus pauvres est paradoxal (Le Bras, 2017 : 49).

Dans ce sens, le désir du commandant de partir et de devenir une des «ombres de Massambalo » (Gaudé, 2006 : 234) reste pourtant ambigu : il meurt en ne laissant « rien derrière lui (...) [que] des perles vertes » (Gaudé, 2006 : 238), cadeau offert par le frère malade de Soleiman au moment de leur séparation.

Pour conclure, l'imaginaire exotopique de ces deux auteurs transnationaux permet de mettre en scène des voix multiples, provenant des espaces difficiles, deux portes insulaires de l'Europe. La juxtaposition de voix multiples qui s'entrecroisent est révélatrice d'une autre forme de regard à la fois esthétique et critique qui mérite plus d'attention.

\section{BIBLIOGRAPHIE}

APPANAH, Nathacha (2016). Tropique de la violence. Paris : Gallimard. 
BAUDRILLARD, Jean (1990). La Transparence du Mal : Essai sur les phénomènes extrêmes. Paris : Galilée.

BAKHTINE, Mikhaïl (1984). Esthétique de la création verbale. Paris : Gallimard.

BOSCHET, Adrien \& GUÉGAN, Jean-Baptiste (2017). Comprendre les migrations : Approches géographique et géopolitique. Clamecy : Bréal.

BouRdieu, Pierre (1993). «L'espace des points de vue » in Pierre Bourdieu (éd.). La Misère du monde. Paris : Éditions du Seuil, pp. 13-17.

CHEVALLIER, Jean \& GHEERBRANT, Alain (1982). Dictionnaire des symboles. Paris : Robert Laffont et Éditions Jupiter.

DIOME, Fatou (2017). Marianne porte plainte! Paris : Flammarion.

GAUDÉ, Laurent (2006). Eldorado. Arles : Actes Sud.

HENRY, Michel \& LÉCHENET, Alexandre \& BIG (2015). « Méditerranée : traverser, coûte que coûte » [on-line]. Libération (le 17/6/2015) [disponible le 17/09/2017] <URL: http://www.liberation.fr/ planete/2015/06/17/traverser-coute-que-coute_1331905>.

LAHLOU, Yasmina (2015). « Migrations : Le cimetière marin », Francophonie du Sud, n 36

(septembre - octobre 2015), pp. 2-3.

LE BRAS, Hervé (2017). L'Âge des migrations. Paris : Éditions Autrement.

MCCUSKER, Maeve \& SOARES, Anthony (2011). Islanded Identities: Constructions of Postcolonial Cultural Insularity. Rodopi: New York.

MONTENAY, Yves (2003). Le Mythe du fossé Nord-Sud ou comment on cultive le sous-développement. Paris : Les belles lettres.

SAVIDAN, Patrick (2009). Le Multiculturalisme. Paris : Presses universitaires de France.

SARTRE, Jean-Paul (1946). L'Existentialisme est un humanisme. Paris : Nagel.

\section{NOTES}

1. Voir à ce sujet l'article de Michel Henry et al. qui désignent les routes les plus fréquentées comme "portes de l'Europe-forteresse » qui sont aussi les plus dangereuses: le passage du Sénégal aux Canaries ou encore, le passage par le centre, de Tunisie ou Libye. Chez Gaudé, le personnage Soleiman fait écho de ce fait dramatique lorsqu'il explique ceci : «Et les émigrants continueront à se presser aux portes de l'Europe, toujours plus pauvres, toujours plus affamés » (Gaudé, 2006 : 225).

2. «Les kwassas sanitaires transportent des malades, des vieux, des femmes enceintes, des enfants handicapés, des blessés graves, des fous, des brûlés. Ils font la traversée entre Anjouan et Mayotte pour se faire soigner. J'ai vu des femmes avec des cancers tellement avancés qu'ils n'existent plus, en métropole, que dans les livres de médecine. J'ai vu des grands brûlés à la peau toute pourrie, des bébés morts dans les bras de leurs mères, des hommes aux jambes sectionnées par des requins» (Appanah, 2016:21).

3. «Le bébé a un œil noir et un œil vert. Il est atteint d'hétérochromie, une anomalie génétique absolument bénigne » (idem :23).

4. « Je vais devoir faire confiance à des gens que je ne connais pas. Je ne suis plus qu'une ombre. Juste une ombre qui laisse derrière elle un petit filet de poussière » (Gaudé, 2006 : 98). 
5. «Si Marianne se laisse faire, sa robe de princesse des Lumières sera ternie par ceux de ces enfants qui, aujourd'hui, se moquent des grands d'hier» (Diome, 2017 : 137).

6. Cette vision nous ramène à l'étude de Maeve McCusker et d'Anthony Soares, Islanded Identities (Rodopi, 2011) qui explique qu'il est de nombreuses îles au XXI ${ }^{\mathrm{e}}$ siècle qui sont de véritables laboratoires, soumis à des interventions humanitaires.

\section{RÉSUMÉS}

Cet article s'intéressera à la structure polyphonique dans deux textes transnationaux, Eldorado de Laurent Gaudé (Actes Sud, 2006) et Tropique de la violence de Nathacha Appanah (Gallimard, 2016). Il s'agira d'aborder l'image de deux portes insulaires de l'Europe, Lampedusa en Méditerranée ainsi que Mayotte dans l'océan Indien, à travers les récits de nombreux personnages qui mettent en cause les progrès civilisationnels du Vieux Continent face à la crise migratoire dans deux contrées éloignées sur le plan géographique. Nous montrerons les convergences et divergences entre ces deux espaces qui renvoient à la présence de problèmes tels que des questions d'ordre socio-politique, moral et éthique, loin de l'image rêvée de l'Eldorado, pour finir sur une réflexion de l'acte d'écrire l'Europe, dans un monde globalisé, dans lequel les textes étudiés ici proposent à la fois une façon de contrer la peur de l'autre, mais tout en envisageant des solutions pourtant paradoxales.

This paper will focus on the polyphonic structure of two transnational texts, Eldorado by Laurent Gaudé (Actes Sud, 2006) and Tropique de la violence by Nathacha Appanah (Gallimard, 2016). It will examine the image of two islands that are doorways to Europe, Lampedusa in the Mediterranean Sea as well as Mayotte in the Indian Ocean, through the accounts of multiple characters who question the presence of civilizational progress on the old Continent as it faces a migration crisis in two geographically remote areas. The convergences and divergences between these two spaces point to the existence of problems that are socio-political, moral and ethical in nature, thus far from the dreamed image of the Eldorado. The last section will be a reflection about the act of writing Europe in a globalized world, in which the texts under investigation propose both a way of countering the fear of the other, but at the same time contemplate paradoxical solutions.

\section{INDEX}

Keywords : doorways to Europe, globalization, Indian Ocean, Mediterranean Sea, migration crisis, polyphonic structure

Mots-clés : crise migratoire, Méditerranée, mondialisation, océan Indien, portes de l'Europe, structure polyphonique

\section{AUTEUR}

SILVIA U. BAAGE

McDaniel College

sbaage@gmail.com 